\title{
Modeling of Fine Migration in a Porous Medium
}

\author{
Yuri Galaguz ${ }^{1}$, and Galina Safina ${ }^{1, *}$ \\ ${ }^{1}$ Moscow State University of Civil Engineering, 129337 Moscow, Russia
}

\begin{abstract}
The structure of underground waters and water permeability of rocks must be taken into account when choosing the location of construction objects, the design of tunnels, hydraulic and underground structures. The model of filtration for suspension in solid porous medium during its displacement with clean water is considered. The numerical calculation of boundary of two phases is carried out and concentrations of suspended and retained particles are calculated for different values of porosity and permeability of the porous medium.
\end{abstract}

\section{Introduction}

The structure of underground waters and water permeability of rocks must be taken into account when choosing the location of construction objects, the design of tunnels, hydraulic and underground structures [1]. Underground waters significantly effect the construction properties of soils. Intensive filtration of water flows leads to soil loosening and reducing its strength. Solution of filtration problems allows forecasting flooding of multistory buildings and estimates the long-term sustainability of building structures.

Underground waters passing through the porous rocks and soil transport a lot of small solid particles. The fluid of particles in liquid is called the suspension. Fine migration one-dimensional problem of particle filtration in the solid porous medium during its displacement with pure water is considered $[2,3]$. The pores in the filter form intersecting channels of different lengths and shapes. In this paper the mathematical model of the particles motion in the filter based on the mechanical-geometric interaction of particles with porous medium is considered. Assume that all the particles are solid balls of equal size, and the channels have circular cross-section of constant diameter throughout its length. When constructing the particle diffusion models the influence of fluid viscosity and electrical interaction of the particles with the channel walls are neglected. It is assumed that the solid particles pass freely through the large pores and get stuck in the throat of small pores which are smaller than the particle diameter [4,5]. The retained particles cannot be driven out by the fluid flow or other particles. Deep bed filtration is described by the equation of mass balance of suspended and retained particles of suspension and the kinetic equation for deposit growth [6]. During long-time filtration the number of free small pores is significantly reduced, and the porosity and permeability of the porous medium are changed. To take into account this phenomenon, in contrast to the classical equations of filtration the dependence of coefficients of the mass balance equation on deposit concentration $S(x, t)$ is

\footnotetext{
* Corresponding author: minkinag@mail.ru
} 
introduced [7]. It is assumed that the deposit growth is proportional to the concentration $C(x, t)$ of suspended particles. The proportionality coefficient depends on the deposit concentration and is called the filtration coefficient [8].

The solution of the problem of particle diffusion in the filter describes the process of particles transport through the pores of the filter and the dynamics of precipitation. Exact and asymptotic solutions of filtration problems are found for some important special cases $[6,9-15]$, but in general, the problem does not have an analytical solution.

In problem considered at the initial moment the porous medium is filled with suspension with given concentrations of suspended and retained particles. At the filter inlet the clean water begins to flow. It is gradually displacing the suspension and filling the porous medium. In a part of the porous medium, which is after water front, filtration of the suspension is terminated, the concentration of suspended particles becomes zero, and the concentration of retained particles is constant.

\section{Mathematical model}

In the domain $\Omega=\{0<x<1, t>0\}$ the suspended and retained particles concentrations $C(x, t), S(x, t)$ satisfy the equations

$$
\begin{gathered}
\frac{\partial}{\partial t}(g(S) C)+\frac{\partial}{\partial x}(f(S) C)+\frac{\partial S}{\partial t}=0 \\
\frac{\partial S}{\partial t}=\Lambda(S) C
\end{gathered}
$$

with border and initial conditions

$$
\begin{gathered}
x=0: C=0 ; \\
t=0: C=C_{0}(x), S=S_{0}(x) .
\end{gathered}
$$

Here porosity $g(S)$, permeability $f(S)$, the filter coefficient $\Lambda(S)$ and initial concentrations $C_{0}(x), S_{0}(x)$ are continuous positive functions.

Equations (1), (2) form a quasi-linear hyperbolic system of the first order. The characteristic curve emerging from the origin is the boundary $\Gamma$ of two phases, it divides the domain $\Omega$ into two subdomains $\Omega_{w}$ and $\Omega_{s}$ containing water and suspense, respectively (see Fig. 1). In $\Omega_{w}$ the suspended particles concentration is zero, the retained particles concentration does not depend on time; in $\Omega_{s}$ the concentrations $C(x, t), S(x, t)$ are variable и positive. According to the method of characteristics since the conditions (3) and (4) disagree at the origin, the solution $C(x, t)$ has a strong discontinuity on the boundary $\Gamma$. The solution $S(x, t)$ is continuous in $\Omega$ and has a weak break on the water front $\Gamma$.

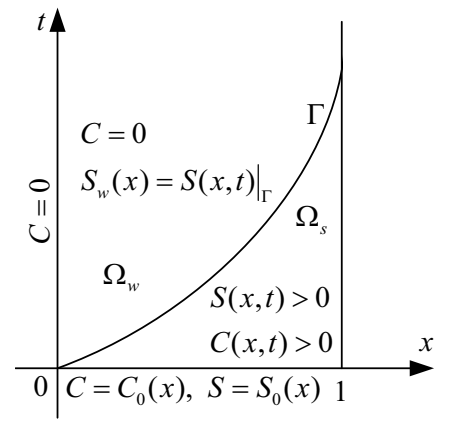

Fig. 1. A schematic solution of the problem (1)-(4). 
If the functions included in the equation (1) are constant and positive

$$
g(S)=g_{0}>0, f(S)=f_{0}>0,
$$

then the boundary $\Gamma$ of two phases and the characteristic of the equation (1) is a straight line

$$
t=\alpha x, \alpha=g_{0} / f_{0} .
$$

For non-permanent functions $g(S), f(S)$ the boundary $\Gamma$ is a curved line, for which there is no analytical expression.

In general, the problem (1)-(4) does not have an analytic solution. The results of numerical calculation by finite differences method as in [16] are given below.

\section{Calculation of fine migration process}

Calculation of boundary of two phases is performed for the filter coefficient $\Lambda(S)=2-S$, porosity $g(S)=1+3 S$ and constant initial conditions $t=0: C=1, S=0.5$ at different values of permeability: $f(S)=1+2 S$ (Fig. 2, a) and $f(S)=1+0.1 S$ (Fig. 2, b).

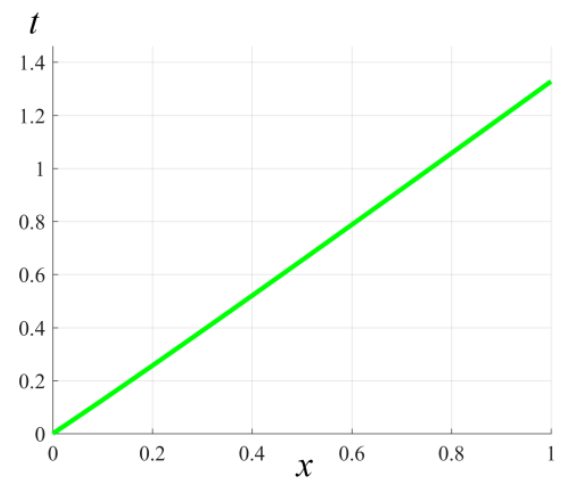

a)

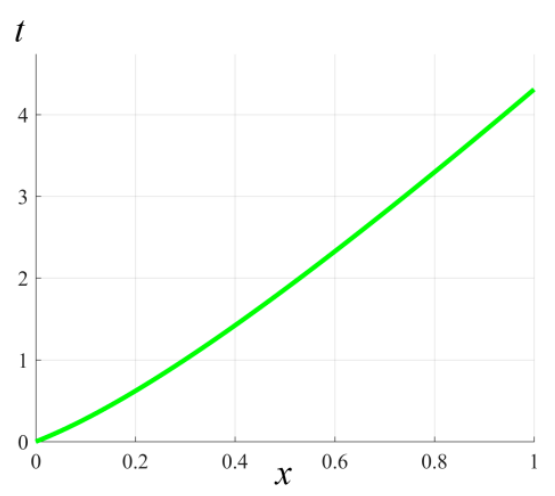

b)

Fig. 2. The boundary between water and suspence.

Calculation of filtration problem is performed by finite difference method for the explicit scheme. The relationship between step $\tau$ in time and step $h$ in coordinate $x$ is selected from the Courant condition $\tau \leq \min _{x, t}(g(S) / f(S)) \cdot h$. However, the best option is reached at $\frac{\tau}{h}=4$, when the numerical solution reflects well the behavior of solutions in discontinuity points. Numerical solution of the problem (1)-(4) is obtained for the coefficients of the equations and constant initial conditions mentioned above for Fig. 2, b) (blue solid line is in Fig. 3-6). Red dotted line represents the solution of the problem for the permanent porosity and permeability $g(S)=f(S)=1$. 
C

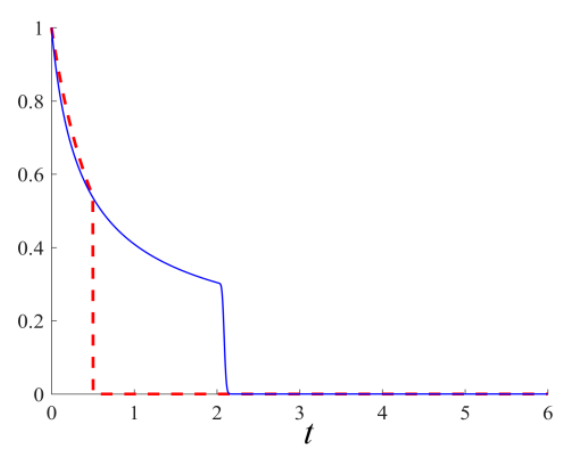

a)

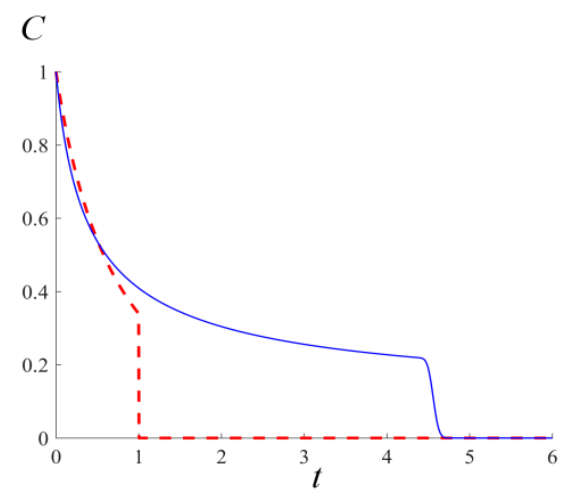

b)

Fig. 3. Suspended particles concentrations for a) $x=0.5$; b) $x=1$.

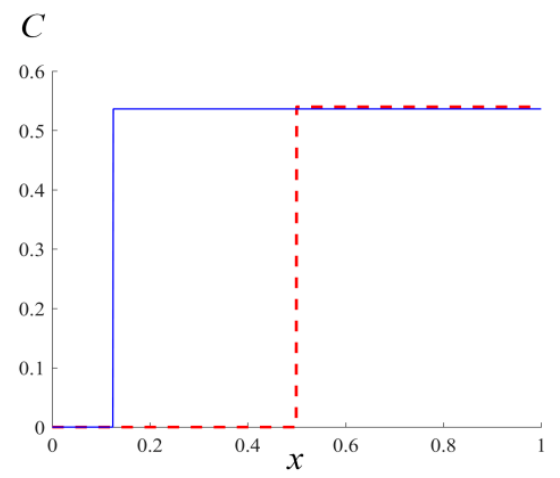

a)

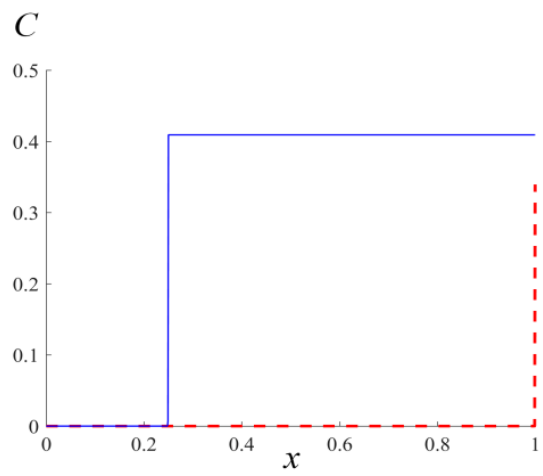

b)

Fig. 4. Suspended particles concentrations for a) $t=0.5$; b) $t=1$.

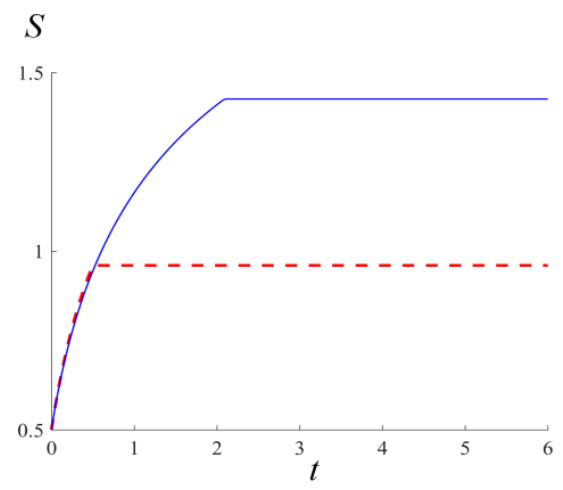

a)

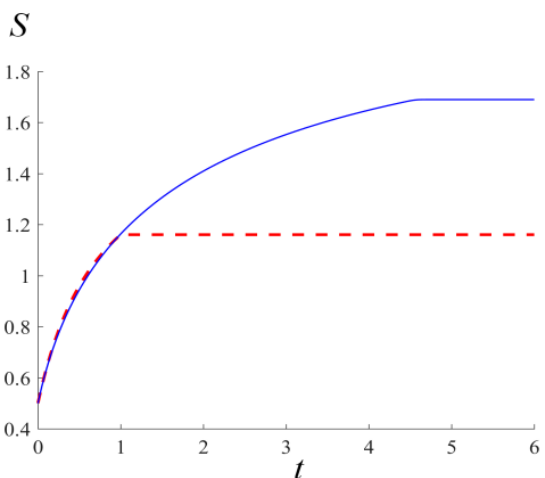

b)

Fig. 5. Retained particles concentrations for a) $x=0.5$; b) $x=1$. 
$S$

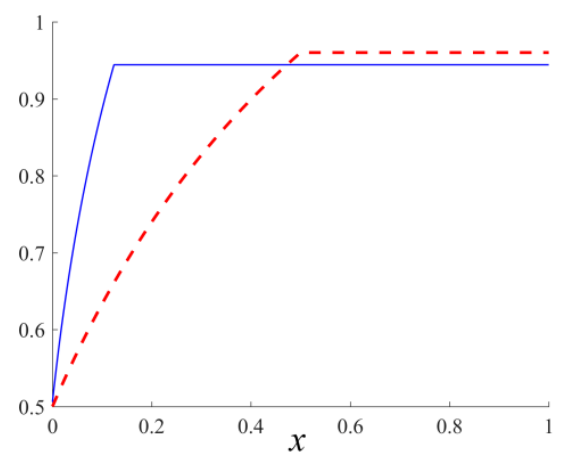

a)

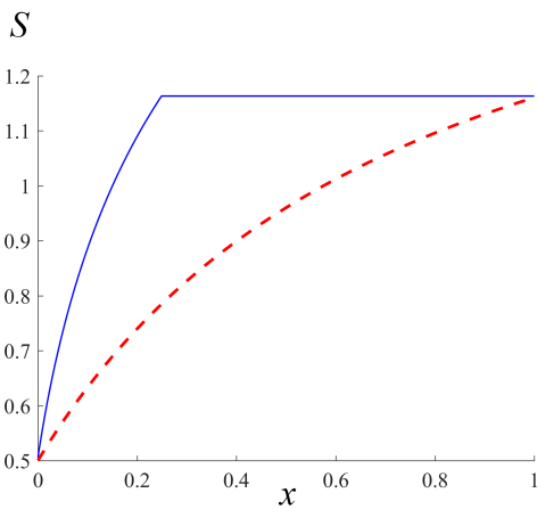

b)

Fig. 6. Retained particles concentrations for a) $t=0.5$; b) $t=1$.

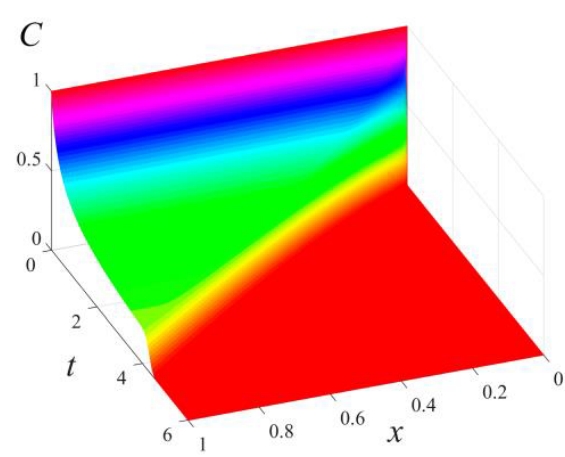

a)

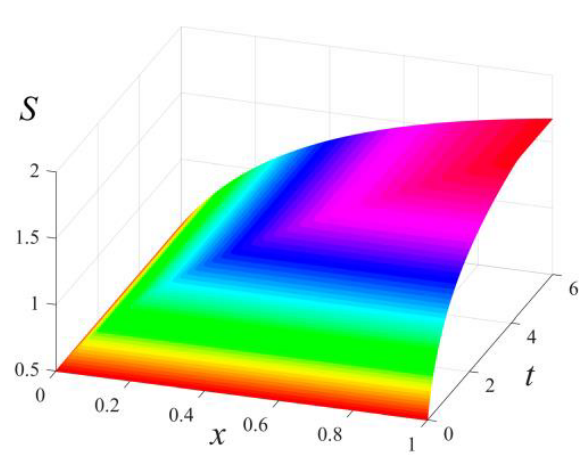

b)

Fig. 7. 3D-graphs a) Suspended particles concentration; b) Retained particles concentration.

\section{Conclusion}

In this paper a numerical solution of the problem of the displacement of a suspension by clean water flow in porous medium is obtained. The boundary of two phases is found, three-dimensional graphics of the concentration of suspended and retained particles and their two-dimensional cross-sections at a fixed time and at a given distance from the filter inlet are constructed.

Calculations show that the boundary of two phases depends on the coefficients of the equations (1) and (2). If $g(S)=f(S)$ then the boundary is a straight line segment. The greater the ratio $g(S) / f(S)$ differs from the constant, the more the curve $\Gamma$ deviates from a straight line.

The introduction of variable porosity and permeability significantly changes the relationship of suspended and retained particles concentrations depending on the time and location. It follows from Fig. 3, 4, 6 that the filtering model with constant functions of porosity and permeability can not serve as a linear approximation of the general non-linear model.

For the numerical calculation the blocking filtration coefficient $\Lambda(S)=2-S$ is selected. In this case for an unlimited time of filtration the retained particles concentration 
$S(x, t) \rightarrow 2$ and the suspended particles concentration $C(x, t) \rightarrow 1$. However water forces out the suspension from a porous medium and prevents particles concentrations to achieve limit values (Fig. 3, 5).

\section{References}

1. K.R.Arora, Soil mechanics and foundation engineering (Delhi, 2004)

2. M.M. Sharma, Y.C. Yortsos, AIChE Journal 33, 10 (1987)

3. K.C. Khilar, H.S. Fogler, Migrations of Fines in Porous Media (Dordrecht, 1998)

4. A. Badalyan, Z. You, K. Aji, P. Bedrikovetsky, T. Carageorgos, A. Rev. Sci. Instrum. 85, ID 015111 (2014)

5. Z. You, A. Badalyan, P. Bedrikovetsky, SPE Journal. 18, 4 (2013)

6. J. P. Herzig, D. M. Leclerc, and P. Legoff, Ind. Eng. Chem. 62, (1970)

7. P. Bedrikovetsky, Transp. in Por. Med. 75, 3 (2008)

8. C. Tien, B.V. Ramarao, Granular filtration of aerosols and hydrosols (Elsevier, Amsterdam, 2007)

9. E.A. Vyazmina, P.G. Bedrikovetskii, A.D. Polyanin, Theor. Found. of Chem. Eng. 41, 5 (2007)

10. Z. You, P. Bedrikovetsky, L. Kuzmina, Abs. and App. Anal., 680693 (2013)

11. Z. You, Y. Osipov, P. Bedrikovetsky, L. Kuzmina, Chem. Eng. J., 258 (2014)

12. L.I. Kuzmina, Yu.V. Osipov, Proc. Eng. 111, (2015)

13. L.I. Kuzmina, Yu.V. Osipov, Proc. Eng. 153, (2016)

14. L.I. Kuzmina, Yu.V. Osipov, Vestnik MGSU. 1, (2015)

15. L.I. Kuzmina, Yu.V. Osipov, Int. J. for Comp. Civ. and Str. Eng. 11, 1 (2015)

16. Yu.P. Galaguz, G.L. Safina, Proc. Eng. 153, (2016) 